\title{
How complex is quantum motion?
}

\author{
Giuliano Benenti ${ }^{1,2}$ and Giulio Casati ${ }^{1,2,3}$ \\ ${ }^{1}$ CNISM, CNR-INFM, and Center for Nonlinear and Complex Systems, Università degli Studi dell'Insubria, Via Valleggio 11, \\ 22100 Como, Italy \\ ${ }^{2}$ Istituto Nazionale di Fisica Nucleare, Sezione di Milano, Via Celoria 16, 20133 Milano, Italy \\ ${ }^{3}$ Centre for Quantum Technologies, National University of Singapore, Singapore 117543
}

(Received 25 July 2008; revised manuscript received 15 January 2009; published 24 February 2009)

\begin{abstract}
In classical mechanics the complexity of a dynamical system is characterized by the rate of local exponential instability which effaces the memory of initial conditions and leads to practical irreversibility. In striking contrast, quantum mechanics appears to exhibit strong memory of the initial state. Here we introduce a notion of complexity for a quantum system and relate it to its stability and reversibility properties.
\end{abstract}

The question of how complex is quantum motion is of fundamental importance with deep connections to entanglement and decoherence. However, our knowledge of the relations between complexity, dynamical stability, reversibility, and chaos is far from being satisfactory, sometimes even confusing, and a clearer understanding is necessary.

To this end let us first consider classical motion where things are quite well settled. Classical complex systems are characterized by positive Lyapunov exponent, i.e., by local exponential instability. They have positive algorithmic complexity and, in terms of the symbolic dynamical description, almost all orbits are random and unpredictable [1].

In spite of many efforts [2], the problem of characterizing the complexity of a quantum system is still open. Indeed the above notion of complexity cannot be transferred, sic et simpliciter, to quantum mechanics, where there is no notion of trajectories. Still, a comparison between classical and quantum dynamics can be made by studying the evolution in time of the classical and quantum phase-space distributions, both ruled by linear equations.

First investigations focused on reversibility, namely, on the propagation of roundoff errors in numerical simulations [3]. Strong and impressive evidence has been gathered that the quantum evolution is very stable, in sharp contrast with classical dynamics in which the extreme sensitivity to initial conditions, which is the very essence of classical chaos, leads to a rapid loss of memory.

Later on, a different approach focused on the stability properties of motion under small variations of system parameters. This approach does not raise any difficulty in the classical context since exponentially unstable systems exhibit the same rate of exponential instability by slightly changing the initial conditions with fixed parameters or by changing parameters with fixed initial conditions. On the other hand, the advantage of the latter approach is that it can be applied to phase-space distributions. Here one computes the so-called fidelity $[4,5]$, defined as the overlap between two distributions evolving under two slightly different Hamiltonians. It is tempting to connect the behavior of fidelity to the regular or chaotic behavior of quantum motion. Indeed, the original expectation, which seemed quite natural, was that fidelity should remain close to 1 at all times for integrable systems and fall down exponentially for chaotic systems $[6,7]$. However, this expectation is not fulfilled.
For the purpose of the present paper it is necessary here to make clear the following. The motivation for the introduction of fidelity, namely, the suggestion to analyze the stability of motion by perturbing the Hamiltonian rather than the initial state, originated from the observation that in quantum mechanics, due to the unitary evolution, the scalar product $\left\langle\psi(t) \mid \psi^{\prime}(t)\right\rangle$ of two initially close states $|\psi(0)\rangle$ and $\left|\psi^{\prime}(0)\right\rangle$ does not change in time. However, two points must be stressed: (i) the classical evolution of phase-space density is also unitary and linear and therefore the overlap of two initially close phase-space distributions does not change with time in classical mechanics as well; (ii) for classically chaotic quantum systems, the fidelity decay, depending on the perturbation strength, can be Gaussian or exponential. A power-law decay is also possible in the quantum diffusive regime [8]. Furthermore, for integrable systems the fidelity decay can be faster than for chaotic systems [5]. On the other hand, even in classical mechanics the fidelity decay does not clearly distinguish between chaotic and integrable systems. In short, fidelity is not a good quantity to characterize the complexity of motion, either in quantum or in classical mechanics.

In this paper, we propose the number of harmonics of the Wigner function as a suitable measure of the complexity of a quantum state. We recall that in classical mechanics the number of harmonics of the classical distribution function in phase space grows linearly for integrable systems and exponentially for chaotic systems, with the growth rate related to the rate of local exponential instability of classical motion [9]. Thus the growth rate of the number of harmonics is a measure of classical complexity. Since the phase-space approach can be equally used for both classical and quantum mechanics, the number of harmonics of the Wigner function appears as the correct quantity to measure the complexity of a quantum state. In what follows, we examine the behavior of this quantity and its relation to fidelity and reversibility properties. Moreover, we show that the number of harmonics can be used to detect the transition from integrability to quantum chaos. A detailed derivation of some of the results discussed in this paper can be found in Ref. [10].

The Wigner function. Let us consider a generic nonlinear system which exhibits a transition from quasi-integrable to chaotic behavior as the strength of the nonlinearity is increased. More precisely we consider the Hamiltonian opera- 
tor $\hat{H} \equiv H\left(\hat{a}^{\dagger}, \hat{a} ; t\right)=H^{(0)}\left(\hat{n}=\hat{a}^{\dagger} \hat{a}\right)+H^{(1)}\left(\hat{a}^{\dagger}, \hat{a} ; t\right)$ with a timeindependent unperturbed part $\hat{H}^{(0)}$ with a discrete energy spectrum bounded from below. Here $\hat{a}^{\dagger}, \hat{a}$ are the bosonic, creation-annihilation operators and $\left[\hat{a}, \hat{a}^{\dagger}\right]=1$.

We will use the method of $c$-number $\alpha$-phase space borrowed from quantum optics (see, for example, [11]). This method is basically built upon the basis of the coherent states $|\alpha\rangle$ which are defined by the eigenvalue problem $\hat{a}|\alpha\rangle$ $=\frac{\alpha}{\sqrt{\hbar}}|\alpha\rangle$, where $\alpha$ is a complex variable independent of the effective Planck's constant $\hbar$. An arbitrary coherent state is obtained from the ground state, $|\alpha\rangle=\hat{D}\left(\frac{\alpha}{\sqrt{\hbar}}\right)|0\rangle$, with the help of the unitary displacement operator $\hat{D}(\lambda)=\exp \left(\lambda \hat{a}^{\dagger}-\lambda^{*} \hat{a}\right)$. The Wigner function $W$ in the $\alpha$-phase plane is related to the density operator $\hat{\rho}$ as follows:

$$
W\left(\alpha^{*}, \alpha ; t\right)=\frac{1}{\pi^{2} \hbar} \int d^{2} \eta e^{\eta^{*}(\alpha / \sqrt{\hbar})-\eta\left(\alpha^{*} / \sqrt{\hbar}\right)} \operatorname{Tr}[\hat{\rho}(t) \hat{D}(\eta)],
$$

where the integration runs over the complex $\eta$ plane.

The harmonic's amplitudes $W_{m}$ of the Wigner function are given by the expansion

$$
W\left(\alpha^{*}, \alpha ; t\right)=\frac{1}{\pi} \sum_{m=-\infty}^{\infty} W_{m}(I ; t) e^{i m \theta}
$$

where $\alpha=\sqrt{I} e^{-i \theta}$, with $(I, \theta)$ action-angle variables.

The fidelity. Following the approach developed in Ref. [12], we consider now the forward evolution

$$
\hat{\rho}(t)=\hat{U}(t) \hat{\rho}(0) \hat{U}^{\dagger}(t)
$$

of an initial (generally mixed) state $\hat{\rho}(0)$ up to some time $t$ $=T$. A perturbation $\hat{P}(\xi)$ is then applied at this time, with perturbation strength $\xi$. For our purposes, it will be sufficient to consider unitary perturbations $\hat{P}(\xi)=e^{-i \xi \hat{V}}$, where $\hat{V}$ is a Hermitian operator. The perturbed state

$$
\hat{\tilde{\rho}}(T, \xi)=\hat{P}(\xi) \hat{\rho}(T) \hat{P}^{\dagger}(\xi)
$$

is then evolved backward, with the same Hamiltonian, for the time $T$, thus obtaining the final state

$$
\hat{\tilde{\rho}}(0 \mid T, \xi)=\hat{U}^{\dagger}(T) \hat{\tilde{\rho}}(T, \xi) \hat{U}(T) .
$$

Finally, we consider the distance between the reversed $\hat{\tilde{\rho}}(0 \mid T, \xi)$ and the initial $\hat{\rho}(0)$ state, as measured by the Peres fidelity [4]

$$
F(\xi ; T)=\frac{\operatorname{Tr}[\hat{\tilde{\rho}}(0 \mid T, \xi) \hat{\rho}(0)]}{\operatorname{Tr}\left[\hat{\rho}^{2}(0)\right]}=\frac{\operatorname{Tr}[\hat{\tilde{\rho}}(T, \xi) \hat{\rho}(T)]}{\operatorname{Tr}\left[\hat{\rho}^{2}(T)\right]} .
$$

This quantity is bounded in the interval $[0,1]$ and the distance between the initial and the time-reversed state is small when $F(\xi ; T)$ is close to 1 . In particular, $F(\xi ; T)=1$ when the two states coincide. The last equality in Eq. (6) is a consequence of the unitary time evolution.

The Peres fidelity (6) can be expressed in terms of the Wigner function as

$$
\begin{aligned}
F(\xi ; T) & =\frac{\int d^{2} \alpha W\left(\alpha^{*}, \alpha ; 0\right) \tilde{W}\left(\alpha^{*}, \alpha ; 0 \mid T, \xi\right)}{\int d^{2} \alpha W^{2}\left(\alpha^{*}, \alpha ; 0\right)} \\
& =\frac{\int d^{2} \alpha W\left(\alpha^{*}, \alpha ; T\right) \tilde{W}\left(\alpha^{*}, \alpha ; T, \xi\right)}{\int d^{2} \alpha W^{2}\left(\alpha^{*}, \alpha ; T\right)} .
\end{aligned}
$$

The advantage of this representation is that it remains valid in the classical case when the Wigner function reduces to the classical phase-space distribution function $W_{c}\left(\alpha^{*}, \alpha ; t\right)$.

Growth of the number of harmonics. As is well known, the paramount property of the classical dynamical chaos is the exponentially fast structuring of the system's phase space on finer and finer scales. In particular, the number $\mathcal{M}(t)$ of harmonics (Fourier components) that significantly contribute in the expansion (2) of the classical phase-space distribution $W_{c}$ grows exponentially in time for chaotic motion. The crucial point is that only in quantum mechanics the number of harmonics of the Wigner function is directly related to the expectation value of physical observables. Therefore, in quantum mechanics an exponential growth of the number of harmonics is not allowed in general $[9,13,14]$.

For an explicit numerical evaluation of the number of harmonics we will focus on the quantity

$$
\left\langle m^{2}\right\rangle_{t}=\frac{\sum_{m=-\infty}^{+\infty} m^{2} \int_{0}^{\infty} d I\left|W_{m}(I ; t)\right|^{2}}{\sum_{m=-\infty}^{+\infty} \int_{0}^{\infty} d I\left|W_{m}(I ; t)\right|^{2}} .
$$

The quantity $\sqrt{\left\langle m^{2}\right\rangle}$ provides an estimate of the number $\mathcal{M}(t)$ of harmonics developed by the time $t$ and therefore of the complexity of the Wigner function at time $t$.

Relation between fidelity and number of harmonics. This relation takes a very simple form when the perturbation at the reversal time $t=T$ is a rotation of the quantum phasespace distribution $W\left(\alpha^{*}, \alpha ; t\right)$. Consider the unitary transformation (3) with the perturbation operator $\hat{V}=\hat{n}$ and the rotation angle $\xi$. In this case we obtain [10]

$$
F(\xi ; t)=1-2 \frac{\sum_{m=-\infty}^{+\infty} \sin ^{2}(\xi m / 2) \int_{0}^{\infty} d I\left|W_{m}(I ; t)\right|^{2}}{\sum_{m=-\infty}^{+\infty} \int_{0}^{\infty} d I\left|W_{m}(I ; t)\right|^{2}} .
$$

The lowest-order $\xi$ expansion of this equation reads

$$
F(\xi ; t) \approx 1-\frac{1}{2} \xi^{2}\left\langle m^{2}\right\rangle_{t}
$$

Notice that relations (9) and (10) between distance $F$ and number of harmonics can be applied to classical dynamics, 


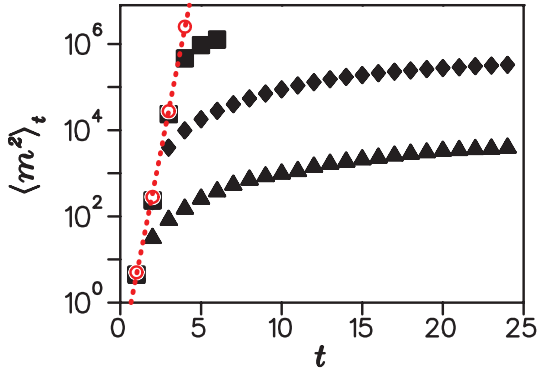

FIG. 1. (Color online) Root-mean-square radius $\left\langle m^{2}\right\rangle_{t}$ of the distribution of harmonics vs time $t$, at $\omega_{0}=1, g_{0}=1.5, \delta=0.5$. Squares, diamonds, and triangles correspond to $\hbar=0.01,0.1$, and 1 . In this latter case, $\hat{\rho}(0)=|0\rangle\langle 0|$. Empty circles refer to classical dynamics and the dashed line fits these data.

provided that the harmonics of the classical distribution function $W_{c}$ instead of those of the Wigner function $W$ are used.

Illustrative example. Let us consider the kicked quartic oscillator model, defined by the Hamiltonian [13,15-17]

$$
\hat{H}=\hbar \omega_{0} \hat{n}+\hbar^{2} \hat{n}^{2}-\sqrt{\hbar} g(t)\left(\hat{a}+\hat{a}^{\dagger}\right),
$$

where $g(t)=g_{0} \Sigma_{s} \delta(t-s), \hat{n}=\hat{a}^{\dagger} \hat{a},\left[\hat{a}, \hat{a}^{\dagger}\right]=1$. In our units, the time and parameters $\hbar, \omega_{0}$ as well as the strength $g_{0}$ of the driving force are dimensionless. The period of the driving force is set to 1 . The corresponding classical Hamiltonian function, expressed in terms of complex canonical variables $\alpha, i \alpha^{*}$, is given by

$$
H_{c}=\omega_{0}|\alpha|^{2}+|\alpha|^{4}-g(t)\left(\alpha^{*}+\alpha\right) .
$$

We choose the initial state to be an isotropic mixture of coherent states: $\hat{\rho}(0)=\int d^{2} \alpha \mathcal{P}\left(|\alpha|^{2}\right)|\alpha\rangle\langle\alpha|$, where $\mathcal{P}\left(I=|\alpha|^{2}\right)$ $=\frac{1}{\pi \Delta} e^{-I / \Delta}$. Correspondingly, the initial Wigner function is isotropic and Gaussian, $W\left(\alpha^{*}, \alpha ; 0\right) \propto e^{-|\alpha|^{2} /(\Delta+\hbar / 2)}$. Only the $m$ $=0$ harmonic is excited, so that $\left\langle m^{2}\right\rangle_{t=0}=0$. The particular case $\Delta=0$ corresponds to the pure ground state, which occupies the minimal quantum cell with the area $\hbar / 2$.

The classical dynamics of model (11) becomes chaotic (with negligible stability islands) when the perturbation strength $g_{0} \gtrsim 1$. In this regime the mean action grows diffusively with the diffusion coefficient $D \approx g_{0}^{2}$.

Chaotic regime. We now compare, in the chaotic regime, the evolution in time of $\left\langle m^{2}\right\rangle_{t}$ for quantum and classical dynamics. For this purpose, we solve both the quantum and the classical Liouville equation. In the latter case, the initial phase-space distribution $W_{c}\left(\alpha^{*}, \alpha ; 0\right) \propto e^{-|\alpha|^{2} / \delta}$ has size $\delta$ which coincides, for a given value of $\hbar$, with the size $\hbar / 2$ of the Wigner function corresponding to the initial quantum ground state $\hat{\rho}(0)=|0\rangle\langle 0|$. The quantum to classical transition is then explored by keeping $\delta$ constant and considering, for smaller and smaller values of $\hbar$, initial incoherent mixtures of size $\delta=\Delta+\hbar / 2$. The results are shown in Fig. 1. The exponential increase of $\left\langle m^{2}\right\rangle_{t}$ takes place only up to the Ehrenfest time scale $t_{E} \propto \ln \hbar[15]$. Note that the short-time correspondence between quantum and classical evolution of the number of harmonics was reported in Refs. $[9,14]$. After that time, a much slower power-law increase follows. Namely,

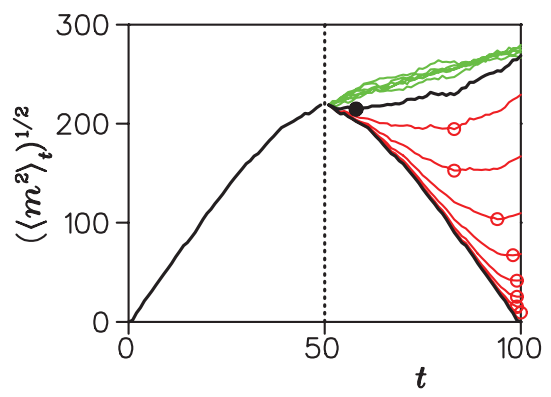

FIG. 2. (Color online) Reversibility properties of quantum dynamics. The backward evolution starts at the reversal time $T=50$. We show $\sqrt{\left\langle m^{2}\right\rangle_{t}}$ for different values of the perturbation parameter: from bottom to top, $\xi=\xi_{c}(T) \times \exp (-l / 2), l=8, \ldots, 1, l=0$ (thick black curve marked by the closed circle), and $l=-1, \ldots,-6$, at $\omega_{0}$ $=1, \hbar=1, g_{0}=2, \Delta=1$. Circles indicate positions of the minimum on each curve.

the number of harmonics $\mathcal{M}(t) \sim \sqrt{\left\langle m^{2}\right\rangle}$ increases linearly for the pure state case, where $\left\langle m^{2}\right\rangle_{t} \approx\langle n\rangle_{t}^{2} \propto t^{2}$ due to diffusive growth of the mean action, and slower than linearly for mixtures [10]. This growth eventually saturates due to quantum localization [13] of diffusive motion.

From Eq. (10) we can estimate a critical perturbation strength $\xi_{c}(T) \approx \sqrt{2 /\left\langle m^{2}\right\rangle_{T}}$, such that the fidelity $F(\xi ; T)$ remains close to 1 after the backward evolution as long as $\xi$ $\ll \xi_{c}(T)$, whereas reversibility is lost when $\xi \gtrsim \xi_{c}(T)$. This statement is illustrated in Fig. 2 for the number of harmonics, estimated by $\sqrt{\left\langle m^{2}\right\rangle_{t}}$. Therefore we establish a direct connection between complexity of phase-space distribution and degree of reversibility of motion. Due to the strikingly different growth in time of the number of harmonics for classical and quantum chaotic motion, $\xi_{c}(T)$ drops exponentially with $T$ in the classical case and at most linearly in the quantum case (after the Ehrenfest time scale). Therefore our analysis explains the numerically observed [3] much weaker sensitivity of quantum dynamics to perturbations as compared to classical dynamics.

Note that, due to the exponential proliferation of the number of harmonics in classical mechanics, formula (10) is only valid up to a time logarithmically short in the perturbation strength $\xi$. After that time, due to diffusive growth of the mean action, the decay of the fidelity $F$ turns from exponential to power law [18], $F \propto 1 /(D t)$, while the number of harmonics still grows exponentially with time and correctly describes the complexity of chaotic motion.

Crossover from integrability to quantum chaos. It is known that in the integrable regime the number of harmonics, computed in the action-angle representation, grows linearly with time [9] and that nearby orbits separate linearly fast [19]. We show (see the inset of Fig. 3) that the linear growth of $\sqrt{\left\langle m^{2}\right\rangle}$ also takes place in quantum mechanics, up to the Heisenberg time scale $t_{H} \propto \hbar^{-1}$. The strikingly different behavior of the number of harmonics of the Wigner function in the integrable and chaotic regimes suggests that this quantity may be used to detect, in the time domain, the crossover from integrability to quantum chaos. Note that the vast literature on such transition is based on the statistical distribution of energy levels [7]. Since in the chaotic regime and 


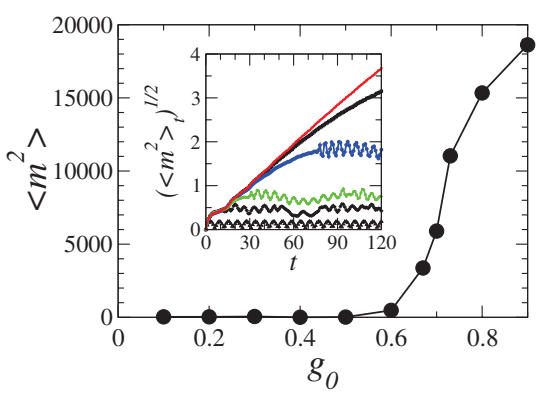

FIG. 3. (Color online) Dependence of $\left\langle m^{2}\right\rangle_{t}$ on the perturbation strength $g_{0}$ at time $t=3$, for $\hbar=0.01, \omega_{0}=1, \Delta=0$. Inset: time evolution of $\sqrt{\left\langle m^{2}\right\rangle_{t}}$ in the integrable regime, at $g_{0}=1, \omega_{0}=1, \delta=0.5$ and, from bottom to top, $\hbar=1,0.1,0.05,0.02,0.01,0.005$.

before the Ehrenfest time, exponential instability leads to an exponential growth of the number of harmonics of the Wigner function, we expect that such quantity can be used to detect, even for times comparable to the Ehrenfest time scale, the integrability to chaos crossover. Indeed, we show in Fig. 3 that $\left\langle m^{2}\right\rangle$, computed at a given time $t$, exhibits a sharp increase when the perturbation parameter $g_{0} \geq 0.5$. From this figure we can conclude that the crossover from integrability to chaos takes place in the region $0.5 \lesssim g_{0}$ $\lesssim 0.7$.

Discussion. To summarize, we have shown that the number of harmonics of the Wigner function is a suitable mea- sure of complexity of a quantum state, in that this quantity is directly related to the reversibility properties of quantum motion and, at the classical limit, reproduces the well-known notion of complexity based on local exponential instability. We would like to stress that in relation to other, very interesting, proposed measures of quantum complexity, such as quantum dynamical entropies [2], our quantity is very convenient for numerical investigations. It becomes therefore possible to investigate complexity as a function of the effective Planck's constant. To establish the proposed different measure of complexity, it will be useful to check it on atypical cases, such as, for instance, integrable systems which exhibit the random matrix theory spectral statistics typical of chaotic systems [20]. Moreover, the above outlined phasespace approach is quite general and can be readily extended to systems with, arbitrary number of degrees of freedom, including qubit systems, whose Hamiltonian can be expressed in terms of a set of bosonic creation-annihilation operators. Therefore in many-body systems the (number of) harmonics of the Wigner function could shed some light on the connection between complexity and entanglement, a fundamental issue of great relevance for the prospects of quantum information science. Moreover, we believe that the dependence of the number of harmonics on control parameters could be used not only to investigate the integrability to quantum chaos crossover but also to detect quantum phase transitions.
[1] J. Ford, Phys. Today 36 (4), 40 (1983); V. M. Alekseev and M. V. Jacobson, Phys. Rep. 75, 287 (1981).

[2] R. Alicki and M. Fannes, Quantum Dynamical Systems (Oxford University Press, New York, 2001); T. Prosen, J. Phys. A 40, 7881 (2007), and references therein.

[3] D. L. Shepelyansky, Physica D 8, 208 (1983); G. Casati, B. V. Chirikov, I. Guarneri, and D. L. Shepelyansky, Phys. Rev. Lett. 56, 2437 (1986)

[4] A. Peres, Phys. Rev. A 30, 1610 (1984).

[5] T. Gorin, T. Prosen, T. H. Seligman, and M. Žnidarič, Phys. Rep. 435, 33 (2006).

[6] A. Peres, Quantum Theory: Concepts and Methods (Kluwer Academic, Dordrecht, 1993).

[7] F. Haake, Quantum Signatures of Chaos, 2nd. ed. (SpringerVerlag, Berlin, 2000).

[8] G. Benenti and G. Casati, Phys. Rev. E 65, 066205 (2002).

[9] A. K. Pattanayak and P. Brumer, Phys. Rev. E 56, 5174 (1997); J. Gong and P. Brumer, Phys. Rev. A 68, 062103 (2003).

[10] V. V. Sokolov, O. V. Zhirov, G. Benenti, and G. Casati, Phys. Rev. E 78, 046212 (2008).
[11] V. Bargmann, Commun. Pure Appl. Math. 14, 187 (1961); R. J. Glauber, Phys. Rev. 131, 2766 (1963); G. S. Agarwal and E. Wolf, Phys. Rev. D 2, 2161 (1970); 2, 2187 (1970).

[12] K. S. Ikeda, in Quantum Chaos: Between Order and Disorder, edited by G. Casati and B. V. Chirikov (Cambridge University Press, 1995).

[13] B. V. Chirikov, F. M. Izrailev, and D. L. Shepelyansky, Sov. Sci. Rev., Sect. C, Math. Phys. Rev. 2, 209 (1981).

[14] Y. Gu, Phys. Lett. A 149, 95 (1990).

[15] G. P. Berman and G. M. Zaslavsky, Physica A 91, 450 (1978); 97, 367 (1979).

[16] V. V. Sokolov, J. Sov. Math. 61, 104 (1985).

[17] V. V. Sokolov, G. Benenti, and G. Casati, Phys. Rev. E 75, 026213 (2007).

[18] G. Benenti, G. Casati, and G. Veble, Phys. Rev. E 67, 055202(R) (2003).

[19] G. Casati, B. V. Chirikov, and J. Ford, Phys. Lett. 77, 91 (1980).

[20] L. Benet, F. Leyvraz, and T. H. Seligman, Phys. Rev. E 68, 045201(R) (2003). 